\title{
TGF- $\beta$ Serum Levels in Diabetic Retinopathy Patients and the Role of Anti-VEGF Therapy
}

\author{
Vincenza Bonfiglio ${ }^{1,+}$, Chiara Bianca Maria Platania ${ }^{2,+}{ }^{+}$, Francesca Lazzara ${ }^{2}$, Federica Conti $^{2}$, \\ Corrado Pizzo ${ }^{3}$, Michele Reibaldi ${ }^{4}{ }^{\circ}$, Andrea Russo ${ }^{3}{ }^{-}$, Matteo Fallico ${ }^{3}{ }^{-0}$, Elina Ortisi ${ }^{3}$, \\ Francesco Pignatelli ${ }^{5}{ }^{\circledR}$, Antonio Longo ${ }^{3}$, Teresio Avitabile ${ }^{3}$, Filippo Drago ${ }^{2,6}$ \\ and Claudio Bucolo ${ }^{2,6, *}$ \\ 1 Department of Experimental Biomedicine and Clinical Neuroscience, Ophthalmology Section, \\ University of Palermo, 90133 Palermo, Italy; enzabonfiglio@gmail.com \\ 2 Department of Biomedical and Biotechnological Sciences, School of Medicine, University of Catania, \\ 95123 Catania, Italy; chiara.platania@unict.it (C.B.M.P.); francesca.lazzara@unict.it (F.L.); \\ conti.federica1@hotmail.it (F.C.); f.drago@unict.it (F.D.) \\ 3 Department of Ophthalmology, University of Catania, 95123 Catania, Italy; corradopizzo@hotmail.it (C.P.); \\ andrearusso2000@hotmail.com (A.R.); matteofallico@hotmail.com (M.F.); elinaortisi@gmail.com (E.O.); \\ ant-longo@libero.it (A.L.); t.avitabile@unict.it (T.A.) \\ 4 Department of Surgical Science, Eye Clinic, University of Torino, 10124 Torino, Italy; mreibaldi@libero.it \\ 5 Department of Ophthalmology, SS Annunziata Hospital, 74121 Taranto, Italy; pignatelli.oculista@gmail.com \\ 6 Center for Research in Ocular Pharmacology-CERFO, University of Catania, 95123 Catania, Italy \\ * Correspondence: claudio.bucolo@unict.it \\ + These authors contributed equally to this work.
}

Received: 24 November 2020; Accepted: 12 December 2020; Published: 15 December 2020

\begin{abstract}
Transforming growth factor $\beta 1$ (TGF $\beta 1$ ) is a proinflammatory cytokine that has been implicated in the pathogenesis of diabetic retinopathy (DR), particularly in the late phase of disease. The aim of the present study was to validate serum TGF $\beta 1$ as a diagnostic and prognostic biomarker of DR stages. Thirty-eight subjects were enrolled and, after diagnosis and evaluation of inclusion and exclusion criteria, were assigned to six groups: (1) healthy age-matched control, (2) diabetic without DR, (3) non-proliferative diabetic retinopathy (NPDR) naive to treatment, (4) NPDR treated with intravitreal (IVT) aflibercept, (5) proliferative diabetic retinopathy (PDR) naïve to treatment and (6) PDR treated with IVT aflibercept. Serum levels of vascular endothelial growth factor A (VEGF-A), placental growth factor (PIGF) and TGF $\beta 1$ were measured by means of enzyme-linked immunosorbent assay (ELISA). Foveal macular thickness (FMT) in enrolled subjects was evaluated by means of structural-optical coherence tomography (S-OCT). VEGF-A serum levels decreased in NPDR and PDR patients treated with aflibercept, compared to naïve DR patients. PlGF serum levels were modulated only in aflibercept-treated NPDR patients. Particularly, TGF $\beta 1$ serum levels were predictive of disease progression from NPDR to PDR. A Multivariate ANOVA analysis (M-ANOVA) was also carried out to assess the effects of fixed factors on glycated hemoglobin (HbA1c) levels, TGF $\beta 1$, and diabetes duration. In conclusion, our data have strengthened the hypothesis that TGF $\beta 1$ would be a biomarker and pharmacological target of diabetic retinopathy.
\end{abstract}

Keywords: diabetic retinopathy; serum biomarkers; anti-VEGFA; TGF $\beta$

\section{Introduction}

Diabetic retinopathy (DR) is a complication of diabetes mellitus, and it is generally defined as the microvascular retinal complication of diabetes [1,2]. DR is clinically classified as non-proliferative (NPDR) and proliferative (PDR). However, several substages have been identified in NPDR patients: 
early, moderate and severe NPDR. The latter is characterized by pervasive retinal hemorrhages and microvascular anomalies [3]. The risk to shift from NPDR to PDR is about $50 \%$; in this perspective, the evaluation of prognosis and correct pharmacological management of NPDR would have a deep impact in the management of DR patients [4]. Diabetic macular edema (DME) is a main microvascular complication of PDR, although it can occur also in severe NPDR [5]. Furthermore, angiogenesis and inflammation are driving factors of DR and DME pathogenesis [6]. Therefore, current DR therapeutical approaches include intravitreal steroids [7] and anti-vascular endothelial growth factor (VEGF) agents, which are generally considered the first-line treatment [8].

Aflibercept is a human recombinant fusion protein that acts as a soluble decoy receptor for VEGF family members, including VEGF-A, VEGF-B and placental growth factor (PIGF) [9,10]. Aflibercept is approved with the following indications: neovascular age-related macular degeneration, macular edema following retinal vein occlusion, diabetic macular edema and diabetic retinopathy [11,12]. Furthermore, aflibercept exerts in vitro and in vivo anti-inflammatory action, modulating the phosphorylation of extracellular signal-regulated kinases (ERK) and decreasing retinal tumor necrosis factor alpha (TNF- $\alpha$ ) release [13]. Besides angiogenesis and inflammation, retinal fibrosis has emerged as a detrimental factor in PDR pathogenesis $[14,15]$. TGF $\beta$ signaling pathway is strictly involved in fibrosis and the remodeling of the extracellular matrix [16,17]; and several reports highlighted that TGF $\beta$ can be implicated in the burden of PDR [18], promoting retinal fibrotic events [15]. Moreover, TGF $\beta$ pathways could promote angiogenesis, along with VEGF $[19,20]$; and TGF $\beta$ isoforms 1-2-3 were reported to induce VEGF expression [20-22].

Most of the retrieved studies reported that TGF $\beta$-signaling activation is detrimental in DR and age-related macular degeneration (AMD) [20-23]. Additionally, some controversial recent data, generated from AMD models, suggested that TGF $\beta$ signaling activation, through TGF $\beta$ receptor 2 (TGF $\beta$ R2), would protect the retina from neuroinflammation and apoptosis, regulating microglia activation and the expression of retinal neurotrophic factors [24-26]. Indeed, the mechanisms underlying TGF $\beta$ pathway activation and retinal neovascularization are complex; furthermore, related data are generally controversial [27]. It has been demonstrated that TGF $\beta$ signaling, through endoglin receptor, promoted subretinal fibro-neovascularization [28]. Shen et al. (2008) demonstrated that anti-VEGF treatment inhibited retinal TGF $\beta$ signaling, reducing $\mathrm{p}$-smad3 levels and leading to decreased inflammation and retinal microglia activation [28]. Intriguingly, recent reports suggested that microRNAs, regulators of angiogenesis and the TGF $\beta$ signaling pathway, would be predictive biomarkers of early phase diabetic retinopathy $[19,29,30]$, along with structural optical coherence tomography (S-OCT) assessment and other clinical outcomes and biomarkers [31].

Therefore, in this pilot study, we tested the hypothesis that the intravitreal injection of aflibercept in DR patients would influence not only the serum levels of VEGF-A and PIGF, but also TGF $\beta 1$. Furthermore, using several statistical analyses (C-statistics of receiver operating characteristics ROC curves and Multivariate-ANOVA), we analyzed the serum levels of VEGF-A and placental growth factor (PIGF), as well as TGF $\beta 1$, to validate novel biomarkers of DR classification and/or new pharmacological targets.

\section{Results}

\subsection{Study Subjects and Ophthalmic Evaluation}

Thirty-eight subjects that fulfilled the eligibility criteria were included in the study (19 males, 19 females, mean age $70 \pm 9$ ) and assigned to six groups (Table 1). Participants' demographics and pre- operative data are reported in Table 1. As regards healthy controls and diabetic, naïve NPDR and PDR patients, fasting venous blood sampling was carried out at the time of the inclusion in this study, after general ophthalmic evaluation and informed consent signature. NPDR and PDR patients that underwent aflibercept treatment were subjected to fasting venous sampling 7 days after intravitreal injection of aflibercept. 
Table 1. Subject demographics.

\begin{tabular}{ccccccc}
\hline & $\begin{array}{c}\text { Gender } \\
\mathbf{( F ;} \mathbf{M})\end{array}$ & $\begin{array}{c}\text { HbA1c } \\
\mathbf{( \% )}\end{array}$ & $\begin{array}{c}\text { Diabetes } \\
\text { Duration } \\
\text { (Years) }\end{array}$ & $\begin{array}{c}\text { Insulin } \\
\text { Treatment } \\
\mathbf{( Y ; ~ N )}\end{array}$ & $\begin{array}{c}\text { Subjects with } \\
\text { Glycemic Control } \\
(\mathbf{Y} ; \mathbf{N})\end{array}$ & $\begin{array}{c}\text { Age } \\
(\text { Years })\end{array}$ \\
\hline CTRL $(\mathrm{N}=7)$ & $(4 ; 3)$ & $3.9 \pm 0.9$ & NA & NA & NA & $66 \pm 14$ \\
\hline Diabetic $(\mathrm{N}=6)$ & $(4 ; 2)$ & $6.6 \pm 0.3$ & $5 \pm 5$ & $(0 ; 6)$ & $(6 ; 0)$ & $75 \pm 10$ \\
\hline NPDR naïve $(\mathrm{N}=6)$ & $(2 ; 4)$ & $7 \pm 1$ & $19 \pm 8$ & $(4 ; 2)$ & $(4 ; 2)$ & $74 \pm 6$ \\
\hline NPDR aflibercept $(\mathrm{N}=6)$ & $(2 ; 4)$ & $6.9 \pm 0.5$ & $20 \pm 8$ & $(5 ; 1)$ & $(2 ; 5)$ & $70 \pm 7$ \\
\hline PDR naïve $(\mathrm{N}=7)$ & $(4 ; 3)$ & $7.3 \pm 0.6$ & $21 \pm 6$ & $(7 ; 0)$ & $(4 ; 2)$ & $67 \pm 8$ \\
\hline PDR aflibercept $(\mathrm{N}=6)$ & $(3 ; 3)$ & $7 \pm 1$ & $18 \pm 9$ & $(3 ; 3)$ & & $70 \pm 7$
\end{tabular}

\subsection{Clinical Assessment}

All diabetic subjects underwent OCT evaluation after study enrollment. Aflibercept-treated eyes (NPDR and PDR) underwent OCT evaluation also 7 days after intravitreal IVT injection (Figure 1). The average OCT foveal macular thickness (FMT) of diabetic patients, without signs of diabetic retinopathy, was $221 \pm 15 \mu \mathrm{m}$. NPDR naïve eyes (no aflibercept IVT treatment) reported a significantly higher OCT macular thickness, $479 \pm 45$ ( $p<0.05)$, compared to diabetic eyes without signs of diabetic retinopathy. PDR naïve patients (no aflibercept IVT treatment) had a significantly higher FMT, $558 \pm 30 \mu \mathrm{m}(p<0.05)$, than values in diabetic and NPDR naïve patients. Either NPDR or PDR patients, after aflibercept treatment, reported a significant decrease of OCT macular thickness $(236 \pm 50$ and $289 \pm 60$, respectively), compared to NPDR and PDR naïve FMT (479 \pm 45 and $558 \pm 30 \mu \mathrm{m}$, respectively), and to the respective baseline values (data not shown, $512 \pm 55$ NPDR pre-treatment, $735 \pm 60$ PDR pre-treatment). After aflibercept treatment, the OCT macular thickness of NPDR patients did not differ from PDR-treated eyes (Figure 1). Furthermore, NPDR and PDR patients, after aflibercept treatment, showed reduced intraretinal cysts. Particularly DR eyes, before treatment, showed an irregular layered structure with flattening of the foveal depression and the presence of large cystoid spaces.

A

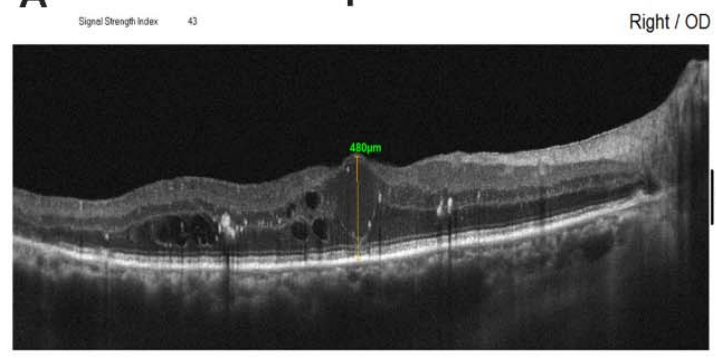

C

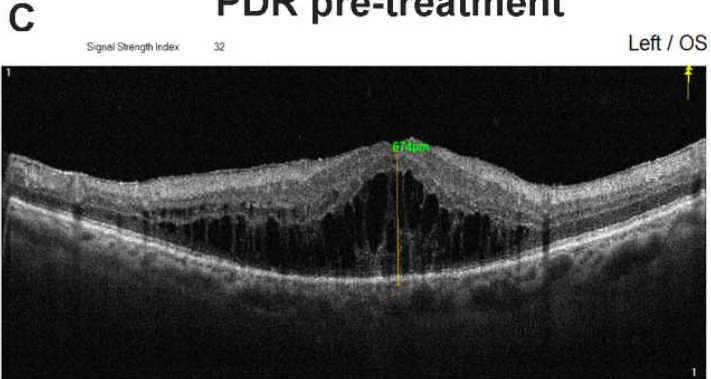

B NPDR post-treatment
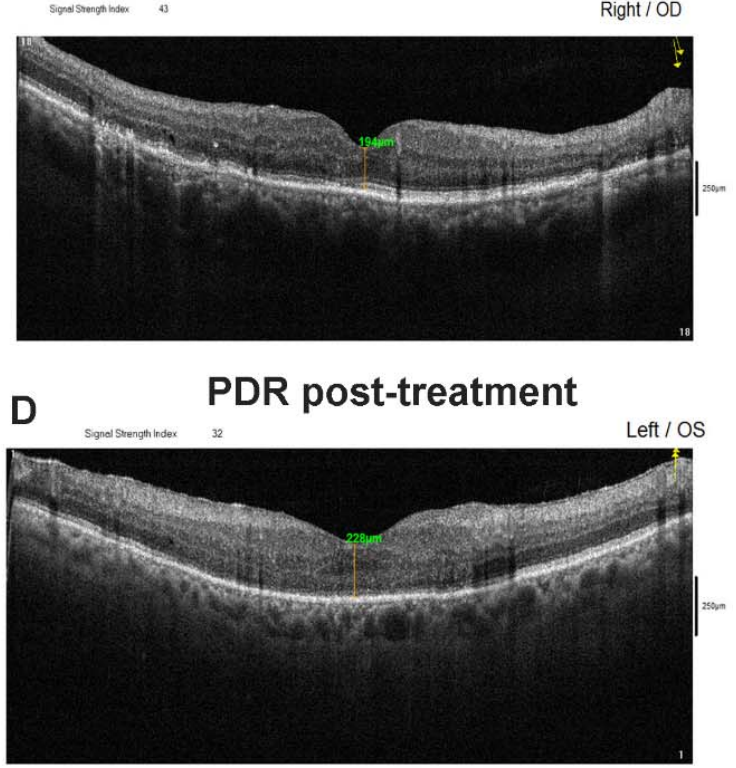

Figure 1. Cont. 

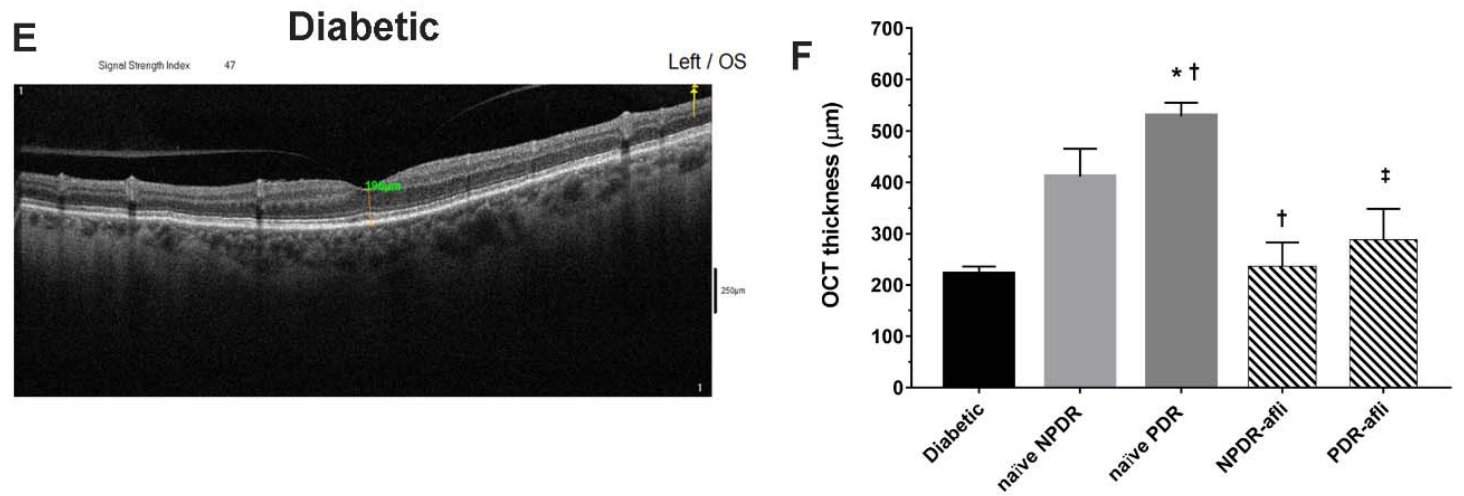

Figure 1. Representative optical coherence tomography (OCT) images of macular thickness. Aflibercept treatment decreased significantly $(p<0.05)$ foveal macular thickness in non-proliferative diabetic retinopathy (NPDR) and proliferative diabetic retinopathy (PDR) patients, compared to untreated naïve groups. Foveal macular thickness measurement in enrolled subjects beloging to the followig groups: NPDR before (A) and after (B) aflibercept treatment, PDR before (C) and after (D) aflibercept treatment, and OCT evaluation in diabetic patients without DR (E). Mean foveal macular thickness (F) $\mu \mathrm{m} \pm$ S.D.; * $p<0.05$ vs. diabetic; $+p<0.05$ vs. NPDR naïve; $\ddagger$ vs. PDR naïve patients.

\subsection{Serum Growth Factor Levels}

Levels of pro-angiogenic factors VEGF-A and PIGF have been evaluated in the serum of the study subjects. VEGF-A serum levels (Figure 2) in diabetic patients, naïve NPDR and PDR subjects, were significantly higher than the levels detected in the serum of age-matched control subjects. The VEGF-A serum levels of NPDR and PDR patients, one week after intravitreal treatment with aflibercept, were significantly decreased when compared to diabetic, naïve NPDR and PDR patients.

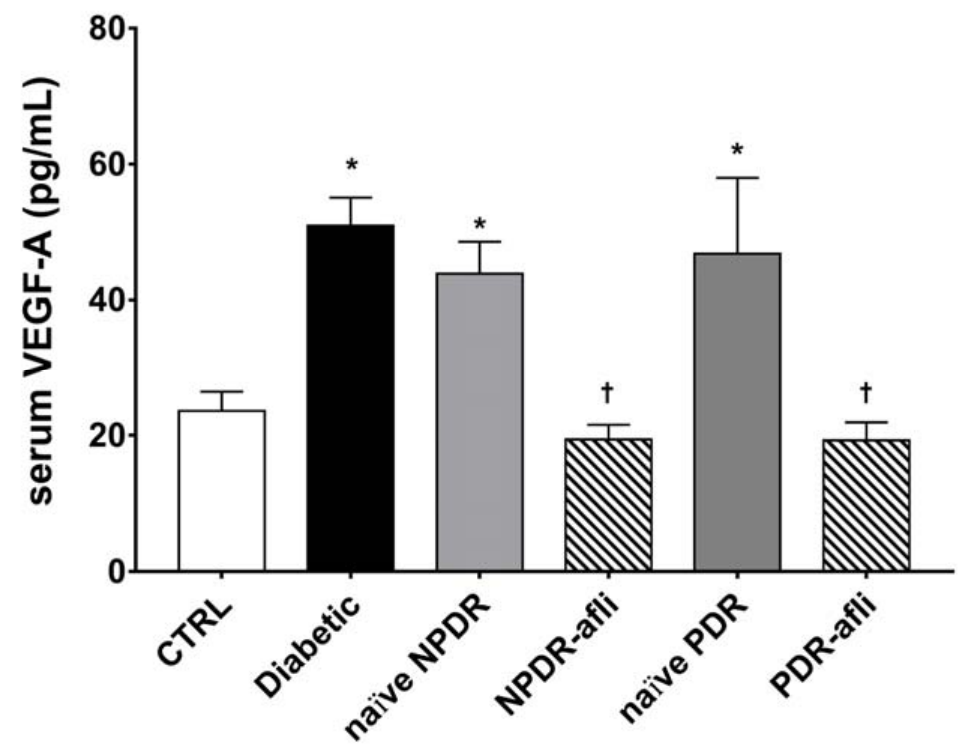

Figure 2. VEGF-A serum levels. After 7 days, aflibercept treatment significantly $(p<0.05)$ decreased VEGFA serum levels in NPDR and PDR patients, compared to diabetic patients without signs of DR, and compared to untreated naïve NPDR and PDR groups. ${ }^{*} p<0.05$ vs. CTRL; † naïve vs. aflibercept (afli) treatment.

Furthermore, we evaluated PlGF levels in the serum of enrolled subjects (Figure 3). PlGF serum levels were higher $(p<0.05)$ in diabetic patients compared to healthy control subjects. No differences were detected between NPDR and PDR naïve patients compared to either control or diabetic patients. One week after aflibercept intravitreal injection, placental growth factor (PIGF) levels in NPDR patients 
were significantly increased $(p<0.05)$ compared to control, diabetic with no DR signs and NPDR naïve patients.

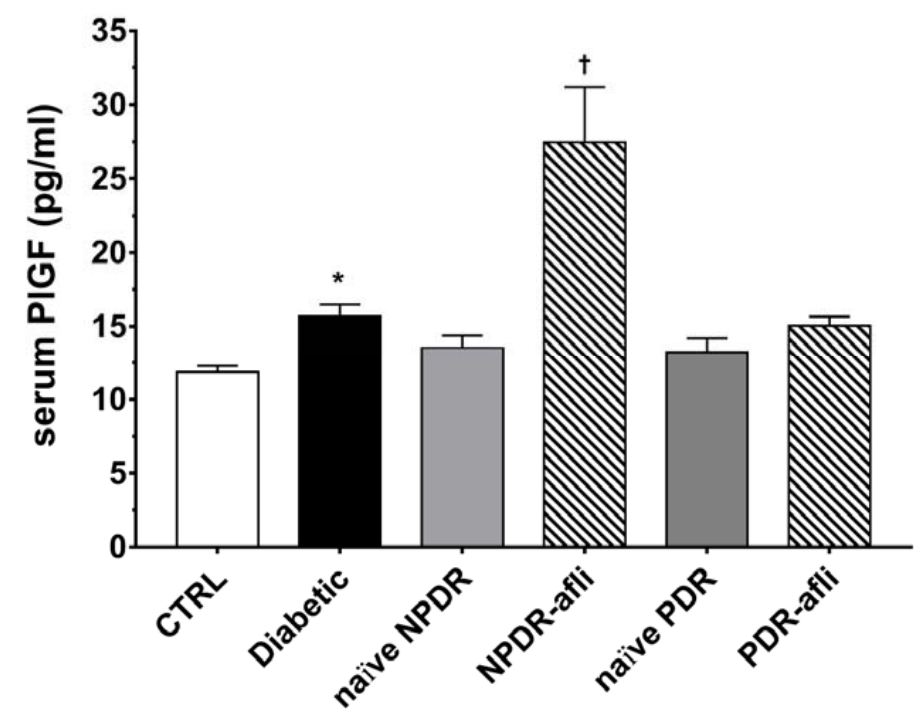

Figure 3. PlGF serum levels. After 7 days, aflibercept significantly $(p<0.05)$ increased PlGF serum levels only in NPDR treated patients, compared to other study subject groups. ${ }^{*} p<0.05$ vs. CTRL; † naïve vs. aflibercept treatment.

TGF $\beta 1$ serum levels (Figure 4$)$ were significantly $(p<0.05)$ higher in the diabetic group, compared to control. Although not significant, TGF $\beta 1$ levels were higher in NPDR naïve patients, compared to diabetic patients without signs of DR. NPDR patients treated with aflibercept, 7 days after the last injection, showed a significant reduction in TGF $\beta 1$ levels, compared to naïve NPDR. PDR patients, either naïve or treated with aflibercept, showed significantly $(p<0.05)$ higher levels of serum TGF $\beta 1$, compared to other study groups.

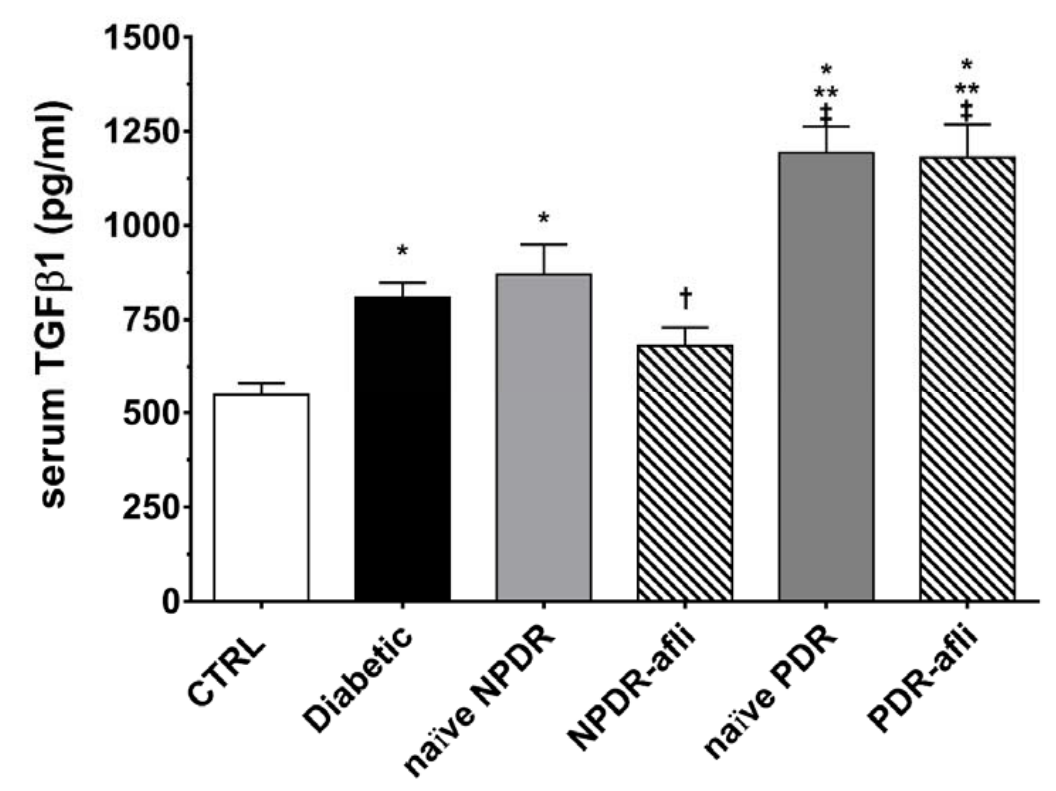

Figure 4. TGF $\beta 1$ serum levels. After 7 days, aflibercept significantly $(p<0.05)$ decreased TGF $\beta 1$ serum levels only in NPDR treated patients, compared to other study subject groups. ${ }^{*} p<0.05 \mathrm{vs}$. CTRL; ** $p<0.05$ vs. diabetic patients without signs of DR; $\uparrow p<0.05$ vs. NPDR naïve patients; $\ddagger p<0.05$ vs. NPDR either naïve or treated patients. 
We aimed at validating TGF $\beta 1$ serum level as a specific and selective biomarker for DR patient stratification (Figure 5). C-statistics revealed that TGF $\beta 1$ levels predicted the classification of: (A). diabetic vs. healthy control patients $(p<0.0001, \mathrm{AUC}=0.94)$; (B). diabetic vs. naïve PDR $(p<0.0001$, AUC $=0.89) ;(C)$. naïve NPDR vs. naïve PDR $(p<0.01, \mathrm{AUC}=0.81) ;(\mathrm{D})$. aflibercept-treated NPDR vs. aflibercept treated PDR patients $(p<0.0001$, AUC $=0.93)$.

A

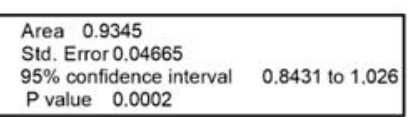

ROC curve: Diabetic vs CTRL

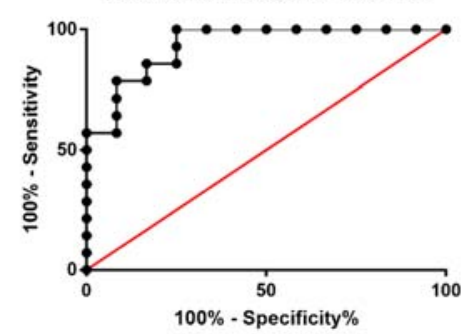

C

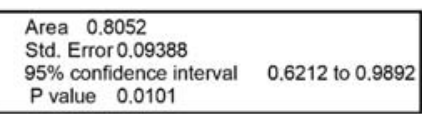

ROC curve: naîve NPDR vs naîve PDR

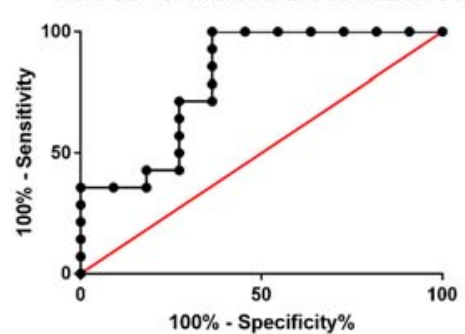

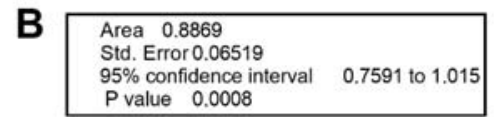

$95 \%$ confidence in
$P$ value 0.0008

ROC curve: Diabetic vs naîve PDR

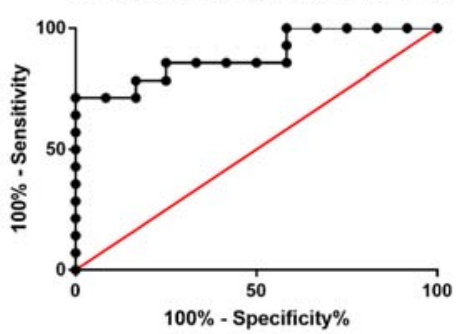

Area 0.9306
Std. Error 0.04953

Std. Error 0.04953
$95 \%$ confidence interval $\quad 0.8335$ to 1.028 $P$ value 0.0003

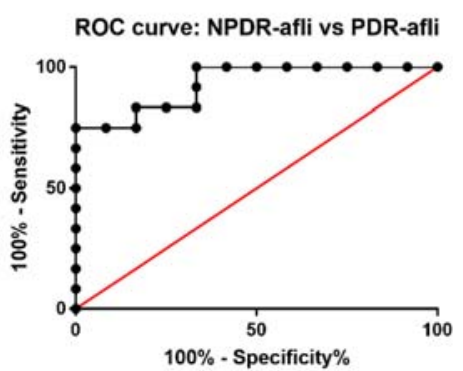

Figure 5. Receiving-operating characteristics curves for TGF $\beta 1$ serum levels. C-statistics validated TGF $\beta 1$ serum levels as a predictive biomarker of (A) diabetic patients without sign of DR (diabetic) compared to control healthy subjects; (B) diabetic compared to naïve PDR patients; (C) naïve NPDR compared to naïve PDR patients; (D) NPDR treated with aflibercept (afli) compared to PDR treated (afli) patients.

Serum TGF $\beta 1$ was not a valid biomarker for the differentiation of NPDR patients from diabetic patients without signs of DR. On the other hand, we validated serum TGF $\beta 1$ as a biomarker of DR progression from the NPDR to the PDR stage (Figure 5).

Furthermore, we carried out a Multivariate ANOVA in order to unveil the effects of fixed factors (independent variables) on dependent variables (diabetes duration, glycated hemoglobin $\mathrm{HbA} 1 \mathrm{c}$, TGF $\beta 1$, VEGFA, PIGF). In this perspective, we checked for the normal distribution of data and Pearson correlation coefficients. We tested with a M-ANOVA the effects of all independent variables only on the duration of diabetes, $\mathrm{HbA} 1$ and TGF $\beta 1$, because these dependent variables were normally distributed (Table 2) and correlated significantly (Table 3). 
Table 2. Normality test.

\begin{tabular}{ccccccc}
\hline & \multicolumn{3}{c}{ Kolmogorov-Smirnov } & \multicolumn{3}{c}{ Shapiro-Wilk } \\
\cline { 2 - 7 } & Statistics & gf & Sign. & Statistics & gf & Sign. \\
\hline Diabetes duration & 0.163 & 38 & 0.014 & 0.926 & 38 & 0.017 \\
\hline HbA1c & 0.190 & 38 & 0.002 & 0.914 & 38 & 0.007 \\
\hline TGFß1 & 0.151 & 38 & 0.033 & 0.937 & 38 & 0.036 \\
\hline VEGF-A & 0.154 & 38 & 0.028 & 0.794 & 38 & 0.000 \\
\hline PlGF & 0.245 & 38 & 0.000 & 0.642 & 38 & 0.000 \\
\hline
\end{tabular}

Table 3. Pearson correlation matrix of dependent variables. ${ }^{* *} p<0.01 ;{ }^{*} p<0.05$. Bold in order to further highlight statistically significant values.

\begin{tabular}{|c|c|c|c|c|c|c|}
\hline & & Duration & HbA1c & TGF $\beta 1$ & VEGF-A & PIGF \\
\hline \multirow{3}{*}{$\begin{array}{l}\text { Diabetes } \\
\text { duration }\end{array}$} & Pearson coefficient & 1 & $0.595 * *$ & $0.335 *$ & 0.102 & 0.084 \\
\hline & Sign. (two tails) & & 0.000 & 0.043 & 0.547 & 0.622 \\
\hline & $\mathrm{N}$ & 38 & 38 & 38 & 38 & 38 \\
\hline \multirow{3}{*}{$\mathrm{HbA1c}$} & Pearson coefficient & $0.595 * *$ & 1 & $0.592 * *$ & 0.271 & 0.163 \\
\hline & Sign. (two tails) & 0.000 & & 0.000 & 0.104 & 0.334 \\
\hline & $\mathrm{N}$ & 38 & 38 & 38 & 38 & 38 \\
\hline \multirow{3}{*}{ TGF $\beta 1$} & Pearson coefficient & $0.335 *$ & $0.592 * *$ & 1 & 0.003 & -0.132 \\
\hline & Sign. (two tails) & 0.043 & 0.000 & & 0.984 & 0.436 \\
\hline & $\mathrm{N}$ & 38 & 38 & 38 & 38 & 38 \\
\hline \multirow{3}{*}{ VEGF-A } & Pearson coefficient & 0.102 & 0.271 & 0.003 & 1 & -0.156 \\
\hline & Sign. (two tails) & 0.547 & 0.104 & 0.984 & & 0.358 \\
\hline & $\mathrm{N}$ & 38 & 38 & 38 & 38 & 38 \\
\hline \multirow{3}{*}{ PlGF } & Pearson coefficient & 0.084 & 0.163 & -0.132 & -0.156 & 1 \\
\hline & Sign. (two tails) & 0.622 & 0.334 & 0.436 & 0.358 & \\
\hline & $\mathrm{N}$ & 38 & 38 & 38 & 38 & 38 \\
\hline
\end{tabular}

The equality of the covariance matrix of dependent variables was satisfied, and the effects of independent variables (group, insulin treatment, glycemic control, gender) on HbA1c, TGF $\beta 1$ and the duration of diabetes were analyzed.

Group, glycemic control and their combinations (group * glycemic control; group * gender; glycemic control * gender) significantly $(p<0.05)$ affected the dependent variables, according to the multivariate analysis of variance (M-ANOVA) (Tables 4 and 5).

Glycemic control significantly affected TGF $\beta 1$ serum levels in patients (Figure 6A). No statistically significant differences were observed between males and females, classified as "good control" or "poor control", according to the provided medical reports (Figure 6A). Furthermore, we found that females showed differences in serum TGF $\beta 1$ levels, compared to males of the same group, although these differences were not always statistically significant (Figure 6B). Particularly, TGF $\beta 1$ levels in females were generally lower compared to males, in most of analyzed groups. On the contrary, females belonging to aflibercept-treated PDR group showed significant $(p<0.05)$ higher levels of TGF $\beta 1$, compared to males. This is because PDR females, treated with aflibercept, had poor glycemic control, and higher $\mathrm{HbA} 1 \mathrm{C}(7.7 \pm 1.1 \%)$ compared to males $(6.7 \pm 1.0 \%)$. 
Table 4. Multivariate test of M-ANOVA. Bold in order to further highlight statistically significant values.

\begin{tabular}{cccc}
\hline Effects & Wilks $\lambda$ & F & $p$-Value \\
\hline group & $\mathbf{0 . 0 3 7}$ & $\mathbf{6 . 8 3 6}$ & $\mathbf{0 . 0 0 0 1}$ \\
\hline insulin treatment & 0.953 & 0.266 & 0.849 \\
\hline glycemic control & $\mathbf{0 . 3 5 8}$ & $\mathbf{0 . 5 7 3}$ & $\mathbf{0 . 0 0 1}$ \\
\hline gender & 0.869 & $0.805 \mathrm{~b}$ & 0.509 \\
\hline Group * glycemic control & $\mathbf{0 . 6 2 7}$ & $\mathbf{3 . 1 6 7}$ & $\mathbf{0 . 0 5}$ \\
\hline Group * gender & $\mathbf{0 . 3 9}$ & $\mathbf{2 . 0 4 9}$ & $\mathbf{0 . 0 5}$ \\
\hline glycemic control * gender & $\mathbf{0 . 4 6 4}$ & $\mathbf{6 . 1 5 2}$ & $\mathbf{0 . 0 0 6}$ \\
\hline
\end{tabular}

Table 5. Between-subjects effects of M-ANOVA. Bold in order to further highlight statistically significant values.

\begin{tabular}{|c|c|c|c|}
\hline Source of Variation & Dependent Variable & $\mathbf{F}$ & $p$-Value \\
\hline \multirow[t]{3}{*}{ group } & HbA1c & 9.624 & 0.0001 \\
\hline & TGF $\beta 1$ & 12.708 & 0.0001 \\
\hline & Diabetes duration & 2.077 & 0.116 \\
\hline \multirow[t]{3}{*}{ Insulin treatment } & $\mathrm{HbA1c}$ & 0.35 & 0.562 \\
\hline & TGF $\beta 1$ & 0.272 & 0.608 \\
\hline & Diabetes duration & 0.095 & 0.762 \\
\hline \multirow[t]{3}{*}{ Glycemic control } & HbA1c & 13.579 & 0.002 \\
\hline & TGF $\beta 1$ & 6.873 & 0.017 \\
\hline & Diabetes duration & 4.582 & 0.046 \\
\hline \multirow[t]{3}{*}{ gender } & $\mathrm{HbA1c}$ & 0.486 & 0.494 \\
\hline & tgfbeta1 & 1.998 & 0.175 \\
\hline & Diabetes duration & 0.062 & 0.806 \\
\hline \multirow[t]{3}{*}{ group * glycemic control } & HbA1c & 6.217 & 0.023 \\
\hline & TGF $\beta 1$ & 0.112 & 0.742 \\
\hline & Diabetes duration & 1.523 & 0.233 \\
\hline \multirow[t]{3}{*}{ group * gender } & $\mathrm{HbA1c}$ & 0.337 & 0.799 \\
\hline & TGF $\beta 1$ & 6.253 & 0.004 \\
\hline & Diabetes duration & 1.123 & 0.366 \\
\hline \multirow[t]{3}{*}{ Glycemic control * gender } & $\mathrm{HbA1c}$ & 0.068 & 0.798 \\
\hline & TGF $\beta 1$ & 15,571 & 0.001 \\
\hline & Diabetes duration & 2.478 & 0.133 \\
\hline
\end{tabular}



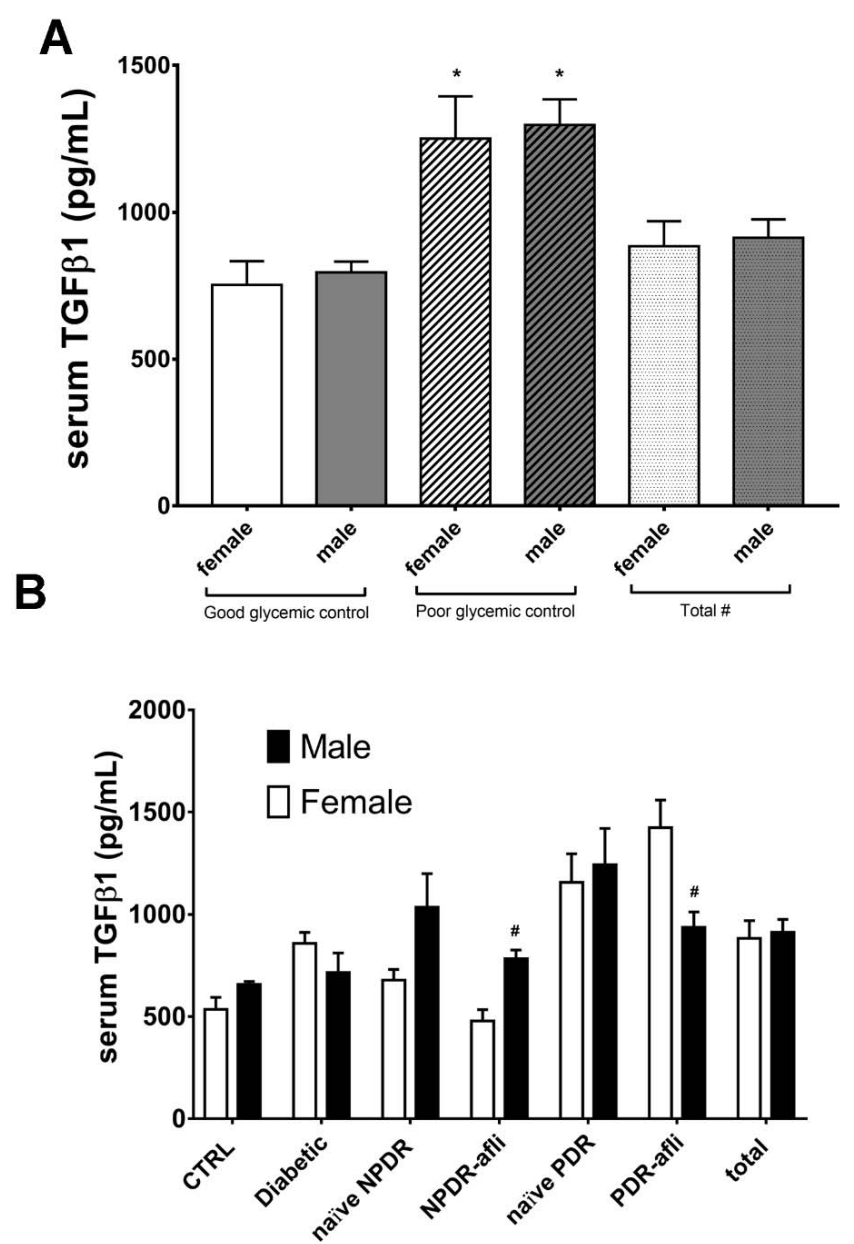

Figure 6. Glycemic control, group and gender effects on TGF $\beta 1$ serum levels. The M-ANOVA post-hoc analysis shed light on fixed factors (independent variables) effects on all analyzed dependent variables. A multivariate-ANOVA guided the stratification of TGF $\beta 1$ levels in terms of glycemic control and gender. (A) Both males and females, with reported poor glycemic control at enrollment, showed significantly $(p<0.05)$ higher levels of TGF1 $\beta$, compared to other patients. The stratification of TGF $\beta 1$ on the basis of gender and subject group (B) showed that only PDR females treated with aflibercept had significantly $(p<0.05)$ higher levels of TGF $\beta 1$, compared to males of the same group. ${ }^{*} p<0.05$ vs. "good glycemic control" group; \# $p<0.05$ males vs. females.

\section{Discussion}

Late diagnosis, duration of diabetes, poor glycemic control and lack of timely/appropriate treatment are the major causes of irreversible vision loss for DR patients [32]. Currently, steroid intravitreal implants/injections [7] and intravitreal injection of anti-VEGF agents are the approved pharmacological treatments of diabetic macular edema, in either non-proliferative (NPDR) or proliferative (PDR) diabetic retinopathy patients [33]. We hereby investigated in a pilot study the clinical outcome (FMT by OCT) and serum cytokines levels (VEGFA, PlGF and TGF 31 ) in six groups of enrolled subjects: healthy controls (age-matched), diabetic without signs of DR, naïve and aflibercept-treated NPDR, naïve and aflibercept-treated PDR patients.

Seven days after the aflibercept IVT injection, FMT decreased significantly $(p<0.05)$ in both NPDR and PDR patients. This result is in accordance with current clinical practice results and with previous reports about anti-VEGF treatment outcomes in severe NPDR patients [34]. OCT foveal macular thickness was slightly higher, although not significantly, in PDR patients, compared to NPDR, after aflibercept treatment, according to previously published reports [35]. Then, we analyzed the VEGF-A and PIGF levels in the serum of all enrolled subjects. Specifically, VEGF-A serum levels 
were significantly $(p<0.05)$ higher in diabetic without signs of DR, NPDR and PDR naïve patients, compared to age-matched healthy subjects. One week after the intravitreal injection of aflibercept, VEGF-A levels decreased significantly in the serum of NPDR and PDR treated patients, compared to other groups. These data are in accordance with the effects of anti-VEGF intravitreal injections on serum VEGF-A, as reported in newborns with retinopathy of prematurity (ROP) [36] or in adults [37]. Moreover, we confirmed that VEGF-A in the serum of DR patients is not predictive of disease staging, as already reported in a previous study [38].

PlGF serum levels were not modified in diabetic patients, compared to controls. It is worthy of note that PIGF levels were significantly increased only in NPDR patients, one week after treatment with aflibercept, and no differences were reported in treated PDR patients compared to controls. This result is in accordance with previous published studies, both in the oncology and ophthalmology areas, describing the increase of serum PIGF as a counter-regulatory mechanism, due to VEGFR2 signaling inhibition by either VEGFR tyrosine kinase inhibitors or anti-VEGF agents [39,40]. Interestingly, the efficacy outcomes generated from the oncological clinical trial VELOUR were not influenced by either VEGF-A or PIGF serum levels, after intravenous injection of aflibercept [41]. Furthermore, in patients with neovascular AMD, PlGF serum levels were found to be increased 7 days after intravitreal injection of aflibercept [42], but authors did not associate the data with an analysis of the clinical outcome. Based on the data of our study, PIGF serum levels were neither predictive of DR staging nor of clinical outcomes. In fact, despite high PIGF serum levels in NPDR patients, the OCT showed a significant decrease of macular edema after aflibercept treatment. On the contrary, PIGF serum levels in PDR patients did not change 7 days after intravitreal injection of aflibercept. This result is probably related to uncontrolled retinal neovascularization in PDR patients, characterized by sustained VEGF signaling that, even if inhibited by an anti-VEGF, would mask any counter-regulatory expression of PIGF, that was observed in NPDR patients.

Serum levels of VEGFA and PIGF were not predictive of DR staging. On the other hand, TGF $\beta 1$ could be considered a sensitive, specific and validated biomarker of DR progression, according to our stratification analysis of subjects. We found significant $C$-statistics of ROC curves for TGF $\beta 1$ serum levels (healthy control vs. diabetic patients, diabetic vs. PDR, naïve NPDR vs. PDR, treated NPDR vs. PDR). On the contrary, on basis of the TGF $\beta 1$ levels, we were not able to differentiate naïve NPDR from diabetic patients without signs of DR. This could be due to limitations of our study, mainly accountable to the limited number of patients, and specifically to the heterogeneity of clinical characteristics of naïve NPDR compared to diabetic patients without signs of DR, which were not treated with insulin and showed low overall duration of diabetes and good glycemic control.

According to a previous study, we found that TGF $\beta 1$ serum levels of DR patients were higher than levels in diabetic group (without DR signs) and control subjects [43]. Furthermore, as regards the quantification of cytokines in sub-silicone oil fluid after vitrectomy, TGF $\beta 1$ levels were significantly higher ( $\sim 3$ fold) in patients with exacerbated PDR, compared to simple PDR (no re-proliferation of fibrotic membrane or vitreous hemorrhage) [44]. Moreover, TGF $\beta 1$ protein was found to be higher also in the aqueous humor of NPDR patients, compared to control subjects [45]. In particular, we observed that NPDR patients, after one week of treatment with aflibercept, showed significantly $(p<0.05)$ reduced serum levels of TGF $\beta 1$ and VEGF-A protein, while the PIGF protein amount was higher compared to that of naïve NPDR patients. These data could be indicative of aflibercept efficacy in NPDR patients. The analysis of the serum of PDR patients, treated with intravitreal injection of aflibercept, highlighted that only VEGFA levels were modified, along with the resolution of the macular edema. On the contrary, TGF $\beta 1$ levels were not significantly modified in PDR treated patients, compared to naïve PDR, possibly due to the clinical and demographic factors on the analyzed population.

Based on this assumption, we carried out a multivariate ANOVA analysis (M-ANOVA), which showed that the diagnosis group, glycemic control and gender (independent variables) influenced TGF $\beta 1, \mathrm{HbA1c}$ and duration of diabetes (dependent variables). This analysis sheds light on the lack of statistically significant differences in TGF $\beta 1$ serum levels between naïve and aflibercept-treated PDR 
patients. In fact, the M-ANOVA analysis highlighted three outliers, bearing high TGF $\beta 1$ serum levels, in aflibercept-treated PDR group: i.e., females with poor glycemic control and higher $\mathrm{HbA1c}$ levels, compared to males. However, based on the present data, we cannot assert that gender influenced TGF $\beta 1$ serum levels and possibly a poor clinical outcome in aflibercept-treated PDR patients. We retrieved a recent pre-clinical report [46] that investigated the effects of sex difference on nephropathy in diabetic mice. This study showed higher renal TGF $\beta 1$ expression levels in female mice [46]. Sex hormones are reported to influence TGF $\beta 1$ [47], while in diabetes mellitus sex differences were found to be related to onset and duration of diabetes, glycemic control, puberty and menopause. In our study all females were in menopause age (see Table 1 reporting subject mean age), therefore we can conclude that gender effects retrieved with M-ANOVA in PDR-treated patients were outliers; i.e., females reporting poor glycemic control and higher $\mathrm{HbA1c}$ levels. However, a big longitudinal study would highlight gender effects on diabetic retinopathy.

Indeed, clinicians should strictly consider DR as a complication of diabetes, warranting a strict management of metabolic clinical outcomes. In this perspective, ophthalmologists should recommend to DR patients a correct management of glycemia and rigid compliance with diabetes therapy $[48,49]$. Furthermore, in DR management it would be useful to monitor not only the macular edema and retinal fundus, but also clinical laboratory parameters such as $\mathrm{HbA1c}$, and possibly TGF $\beta 1$ serum levels. The main drawback of our study is the limited number of patients in each group, and a bigger longitudinal study would strengthen our data and the conclusions regarding the prognostic value of TGF $\beta 1$ in diabetic retinopathy.

In conclusion, TGF $\beta 1$ serum level can be considered a predictive biomarker of disease progression from NPDR to PDR, and it would likely be a secondary endpoint of anti-VEGF clinical efficacy, along with VEGF-A levels. Finally, TGF $\beta 1$ levels correlated with $\mathrm{HbA1c}$ levels and duration of diabetes. Indeed, these two variables should be taken into account by ophthalmologists during the clinical management of diabetic retinopathy.

\section{Materials and Methods}

\subsection{Subjects}

Subjects were enrolled at the Eye Clinic of the University of Catania. All subjects (19 males, 19 females, mean age $70 \pm 9$ ) (Table 1), including age-matched control subjects and diabetic patients without signs of DR (diabetic), diabetic patients with PDR and NPDR, read and signed the informed consent before enrollment. The study complied with the Declaration of Helsinki, and the protocol was approved by the Ethics Committee of the University of Catania (Project identification code \#318). Inclusion criteria are hereby enlisted: age $>18$ years, history of diabetes mellitus type 1 or 2 (diabetic patients). Only patients treated with aflibercept in one eye were included.

Exclusion criteria were macular edema not related to DR, recent ocular surgery (within 6 months), presence of epiretinal membranes/vitreomacular traction and incomplete medical records. Subjects were excluded in case of previous diagnosis of other proliferative vascular diseases, inflammatory diseases, and vitreous hemorrhages. Any previous intravitreal treatments, including both anti-VEGF and corticosteroids, were considered as exclusion criteria.

The diagnosis of non-proliferative and proliferative diabetic retinopathy was assessed by fundus examination using binocular ophthalmoscopy and fluorescein angiography.

Center-involving DME (central foveal macular thickness (FMT) $>300 \mu \mathrm{m}$ ) was assessed by Spectral Domain Optical Coherence Tomography (SD-OCT) (Optovue, Freemont, CA, USA; version 2017.1.0.151 AngioVue Phase 7 Software with PAR) using the retina map mode, which covered a $6.0 \times 6.0 \mathrm{~mm}$ area centered at the fovea.

Naïve NPDR and PDR patients with DME received aflibercept intravitreal injection ( $2 \mathrm{mg} / 0.05 \mathrm{~mL}-$ Eylea ${ }^{\circledR}$, Bayer, Leverkusen, Germany) for the first time at the time of diagnosis. All injections were performed under sterile conditions in a surgical setting, after preparation of the conjunctiva using a $5 \%$ 
povidone-iodine solution, topical anesthetic, and positioning of the lid speculum. Ophthalmic clinical evaluation included fundus examination by binocular ophthalmoscopy, fluorescein angiography (FAG) and SD-OCT. All enrolled subjects underwent fasting venous blood sampling. In particular, blood samples from the NPDR and PDR aflibercept-treated group were collected 7 days after intravitreal injections. Serum samples were aliquoted and stored at $-80^{\circ} \mathrm{C}$ until. Serum samples from each subject were collected and masked with two randomly assigned digits (XY) (https://www.randomcodegenerator. $\mathrm{com} /$ ), and the prefixes A-, B- and C- were assigned to each aliquot to be analyzed for VEGF-A, PIGF and TGF $\beta 1$ quantification, respectively.

\subsection{Enzyme-Linked Immunosorbent Assay (ELISA)}

Serum levels of VEGF-A, PIGF and TGF- $\beta 1$ were quantified by ELISA. Commercial ELISA kits: i. RAB0507 Millipore, Saint Louis, USA; ii. OKBB00242 Aviva systems biology, San Diego, CA, USA; iii. ADI-900-155 ENZO Life Science, Farmingdale, NY were used, respectively, for VEGF-A, PIGF and TGF- $\beta 1$ quantification.

To quantify the VEGF-A levels, according to the manufacturer's instructions, standards and samples were added into appropriate wells coated with anti-human VEGF-A, and the plate was incubated for $2.5 \mathrm{~h}$ at room temperature. After washing four times with the appropriate wash solution, the Biotinylated Detection Antibody was added to each well for $1 \mathrm{~h}$ at room temperature. Subsequently, the washing step has been repeated and a horseradish peroxidase (HRP)-streptavidine solution was added to each well for $45 \mathrm{~min}$ at room temperature. After washing again, 3,3' $5,5^{\prime}$-tetramethylbenzidine (TMB) substrate reagent was added for $30 \mathrm{~min}$ at room temperature in the dark. Lastly, Stop Solution has been added, and the absorbance at $450 \mathrm{~nm}$ was read immediately in a plate reader (VariosKan, Thermo Fisher Scientific, Waltham, MA, USA).

In order to quantify PIGF, standards and samples were added in the anti-human PlGF pre-coated well plate and incubated at $37^{\circ}$ for $90 \mathrm{~min}$. After discarding the liquid in the wells, biotinylated anti-human PlGF antibody was added to each well, and the plate was incubated at $37^{\circ}$ for $60 \mathrm{~min}$. Subsequently the plate was washed three times with the specific wash buffer. According to the manufacturer's instructions, Avidin-Biotin-Peroxidase Complex (ABC) was added into each well and incubated at $37^{\circ}$ for $30 \mathrm{~min}$. Then, the washing step was repeated five times, and TMB Color Developing Agent was added to each well for $15-25$ min at $37^{\circ}$ in the dark. Lastly, TMB Stop solution has been added, and the absorbance was read at $450 \mathrm{~nm}$ in a plate reader (VariosKan, Thermo Fisher Scientific, Waltham, MA, USA).

For TGF- $\beta 1$ detection, serum samples were activated by adding $2.5 \mathrm{~N}$ acetic acid/10M urea. After $10 \mathrm{~min}$ of incubation at room temperature, the samples were neutralized with $2.7 \mathrm{~N} \mathrm{NaOH} / 1 \mathrm{M}$ HEPES and Assay Buffer 13 was added, according to the manufacturer's instruction. Activated samples and standards were added for $1 \mathrm{~h}$ at room temperature to wells coated with a human monoclonal antibody specific for TGF- $\beta 1$. After washing four times with the specific Wash Buffer, a yellow solution of polyclonal antibody to TGF- $\beta 1$ was added, and the plate was incubated for $2 \mathrm{~h}$ at room temperature. The plate was washed again to remove excess antibodies. A blue solution of HRP conjugate was added to each well and incubated for $30 \mathrm{~min}$ at room temperature. After washing, TMB substrate solution was added for $30 \mathrm{~min}$ at room temperature. Lastly, after adding Stop Solution, the optical density was read at $450 \mathrm{~nm}$ in a plate reader (VariosKan, Thermo Fisher Scientific, Waltham, MA, USA).

\subsection{Statistical Analysis}

OCT images and the demographic information of enrolled subjects were masked to investigators with random labels, assigned at the time of blood collection and serum sample labeling. Foveal macular thickness analysis, enzyme-linked immunosorbent assay (ELISA) quantification and statistical analysis were carried out by investigators unaware of the groups. The labels were unveiled after raw graph-design and statistical analysis. 
Statistical analyses were carried out with SPSS software, version 21.0 (SPSS Inc., Chicago, IL, USA), and GraphPad Prism (San Diego, CA, USA). The latter software was also used for graph design. Data were presented as mean \pm standard deviation (SD). Parameters were checked for normal distribution, given a $p<0.05$ of the Shapiro-Wilk test. A univariate ANOVA was carried out to test the effects of independent variables (age, gender, insulin treatment, glycemic control) on each single dependent variable (glycated hemoglobin $\mathrm{HbA} 1 \mathrm{c}$, duration of diabetes, TGF $\beta 1$, VEGFA, PIGF). Thereafter, given a significant $\mathrm{F}$ test $(p<0.05)$ and homogeneity of variance, a Tukey-Kramer post-hoc test was carried out for multiple comparison between subject groups. The significance level was set to $p<0.05$. The diagnostic power of biomarkers was evaluated with ROC curves (C-statistics, AUC, confidence interval). Given the normal distribution of data, equality of covariance matrix and significant Pearson correlation for most of the dependent variables (duration of diabetes, $\mathrm{HbA1c}$, TGF $\beta 1$ ), we carried out a multivariate ANOVA (M-ANOVA) to evaluate the effects of independent variables on all dependent variables.

Author Contributions: Conceptualization, V.B., C.B.; validation, C.B.M.P., F.L., F.C., C.P., V.B., C.B., M.R., A.R., M.F., F.P., A.L.; statistical analysis, C.B.M.P.; investigation, C.B.M.P., C.P., V.B., F.L., F.C., M.R., A.R., M.F., E.O., F.P., A.L.; resources, V.B., C.B., F.D. and T.A.; data curation, V.B., C.B.M.P., C.P.; writing- original draft preparation, C.B.M.P., V.B., C.B.; writing-review \& editing, V.B., C.B., C.B.M.P.; supervision, V.B., C.B.; project administration, V.B., T.A., F.D. and C.B.; funding acquisition, V.B. and C.B. All authors have read and agreed to the published version of the manuscript.

Funding: This research received no external funding.

Acknowledgments: University of Catania grant "PIACERI 2020-2022—Linea Intervento 4" - “Fondi di Ateneo 2020-2022-Università di Catania, linea Open Access".

Conflicts of Interest: Authors have no conflict of interest to declare.

\section{References}

1. Miller, D.J.; Cascio, M.A.; Rosca, M.G. Diabetic Retinopathy: The Role of Mitochondria in the Neural Retina and Microvascular Disease. Antioxidants 2020, 9, 905. [CrossRef] [PubMed]

2. Grading Diabetic Retinopathy from Stereoscopic Color Fundus Photographs-An Extension of the Modified Airlie House Classification: ETDRS Report Number 10. Ophthalmology 2020, 98, 786-806. [CrossRef]

3. Parravano, M.; De Geronimo, D.; Scarinci, F.; Querques, L.; Virgili, G.; Simonett, J.M.; Varano, M.; Bandello, F.; Querques, G. Diabetic Microaneurysms Internal Reflectivity on Spectral-Domain Optical Coherence Tomography and Optical Coherence Tomography Angiography Detection. Am. J. Ophthalmol. 2017, 179, 90-96. [CrossRef] [PubMed]

4. Lee, C.S.; Lee, A.Y.; Baughman, D.; Sim, D.; Akelere, T.; Brand, C.; Crabb, D.P.; Denniston, A.K.; Downey, L.; Fitt, A.; et al. The United Kingdom Diabetic Retinopathy Electronic Medical Record Users Group: Report 3: Baseline Retinopathy and Clinical Features Predict Progression of Diabetic Retinopathy. Am. J. Ophthalmol. 2017, 180, 64-71. [CrossRef] [PubMed]

5. Bandello, F.; Cicinelli, M.V. 19th EURETINA Congress Keynote Lecture: Diabetic Retinopathy Today. Ophthalmologica 2020, 243, 163-171. [CrossRef]

6. Romero-Aroca, P.; Baget-Bernaldiz, M.; Pareja-Rios, A.; Lopez-Galvez, M.; Navarro-Gil, R.; Verges, R. Diabetic Macular Edema Pathophysiology: Vasogenic versus Inflammatory. J. Diabetes Res. 2016, 2016, 2156273. [CrossRef]

7. Bucolo, C.; Gozzo, L.; Longo, L.; Mansueto, S.; Vitale, D.C.; Drago, F. Long-term efficacy and safety profile of multiple injections of intravitreal dexamethasone implant to manage diabetic macular edema: A systematic review of real-world studies. J. Pharmacol. Sci. 2018, 138, 219-232. [CrossRef]

8. Moulin, T.A.; Adjei Boakye, E.; Wirth, L.S.; Chen, J.; Burroughs, T.E.; Vollman, D.E. Yearly Treatment Patterns for Patients with Recently Diagnosed Diabetic Macular Edema. Ophthalmol. Retin. 2019, 34, 362-370. [CrossRef]

9. Papadopoulos, N.; Martin, J.; Ruan, Q.; Rafique, A.; Rosconi, M.P.; Shi, E.; Pyles, E.A.; Yancopoulos, G.D.; Stahl, N.; Wiegand, S.J. Binding and neutralization of vascular endothelial growth factor (VEGF) and related ligands by VEGF Trap, ranibizumab and bevacizumab. Angiogenesis 2012, 15, 171-185. [CrossRef] 
10. Platania, C.; Di Paola, L.; Leggio, G.M.; Romano, G.L.; Drago, F.; Salomone, S.; Bucolo, C. Molecular features of interaction between VEGFA and anti-angiogenic drugs used in retinal diseases: A computational approach. Front. Pharmacol. 2015, 6, 248. [CrossRef]

11. Plyukhova, A.A.; Budzinskaya, M.V.; Starostin, K.M.; Rejdak, R.; Bucolo, C.; Reibaldi, M.; Toro, M.D. Comparative Safety of Bevacizumab, Ranibizumab, and Aflibercept for Treatment of Neovascular Age-Related Macular Degeneration (AMD): A Systematic Review and Network Meta-Analysis of Direct Comparative Studies. J. Clin. Med. 2020, 9, 1522. [CrossRef] [PubMed]

12. Avitabile, T.; Azzolini, C.; Bandello, F.; Boscia, F.; De Falco, S.; Fornasari, D.; Lanzetta, P.; Mastropasqua, L.; Midena, E.; Ricci, F.; et al. Aflibercept in the treatment of diabetic macular edema: A review and consensus paper. Eur. J. Ophthalmol. 2017, 27, 627-639. [CrossRef] [PubMed]

13. Lazzara, F.; Fidilio, A.; Platania, C.B.M.; Giurdanella, G.; Salomone, S.; Leggio, G.M.; Tarallo, V.; Cicatiello, V.; De Falco, S.; Eandi, C.M.; et al. Aflibercept regulates retinal inflammation elicited by high glucose via the PIGF/ERK pathway. Biochem. Pharmacol. 2019, 168, 341-351. [CrossRef] [PubMed]

14. Platania, C.B.M.; Leggio, G.M.; Drago, F.; Salomone, S.; Bucolo, C. Computational systems biology approach to identify novel pharmacological targets for diabetic retinopathy. Biochem. Pharmacol. 2018, 158, 13-26. [CrossRef]

15. Gucciardo, E.; Loukovaara, S.; Korhonen, A.; Repo, P.; Martins, B.; Vihinen, H.; Jokitalo, E.; Lehti, K. The microenvironment of proliferative diabetic retinopathy supports lymphatic neovascularization. J. Pathol. 2018, 245, 172-185. [CrossRef]

16. Klaassen, I.; van Geest, R.J.; Kuiper, E.J.; van Noorden, C.J.F.; Schlingemann, R.O. The role of CTGF in diabetic retinopathy. Exp. Eye Res. 2015, 133, 37-48. [CrossRef]

17. Fan, J.; Shen, W.; Lee, S.R.; Mathai, A.E.; Zhang, R.; Xu, G.; Gillies, M.C. Targeting the Notch and TGF- $\beta$ signaling pathways to prevent retinal fibrosis in vitro and in vivo. Theranostics 2020, 10, 7956. [CrossRef]

18. Loukovaara, S.; Robciuc, A.; Holopainen, J.M.; Lehti, K.; Pessi, T.; Liinamaa, J.; Kukkonen, K.T.; Jauhiainen, M.; Koli, K.; Keski-Oja, J.; et al. Ang-2 upregulation correlates with increased levels of MMP-9, VEGF, EPO and TGF $\beta 1$ in diabetic eyes undergoing vitrectomy. Acta Ophthalmol. 2013, 91, 531-539. [CrossRef]

19. Lazzara, F.; Trotta, M.C.; Platania, C.B.M.; D’Amico, M.; Petrillo, F.; Galdiero, M.; Gesualdo, C.; Rossi, S.; Drago, F.; Bucolo, C. Stabilization of HIF-1 $\alpha$ in Human Retinal Endothelial Cells Modulates Expression of miRNAs and Proangiogenic Growth Factors. Front. Pharmacol. 2020, 11, 1063. [CrossRef]

20. Nagineni, C.N.; Samuel, W.; Nagineni, S.; Pardhasaradhi, K.; Wiggert, B.; Detrick, B.; Hooks, J.J. Transforming Growth Factor- $\beta$ Induces Expression of Vascular Endothelial Growth Factor in Human Retinal Pigment Epithelial Cells: Involvement of Mitogen-Activated Protein Kinases. J. Cell. Physiol. 2003, 197, 453-462. [CrossRef]

21. Ferrari, G.; Pintucci, G.; Seghezzi, G.; Hyman, K.; Galloway, A.C.; Mignatti, P. VEGF, a prosurvival factor, acts in concert with TGF- $\beta 1$ to induce endothelial cell apoptosis. Proc. Natl. Acad. Sci. USA 2006, 103, 17260-17265. [CrossRef] [PubMed]

22. Grigsby, J.; Betts, B.; Vidro-Kotchan, E.; Culbert, R.; Tsin, A. A possible role of acrolein in diabetic retinopathy: Involvement of a VEGF/TGF $\beta$ signaling pathway of the retinal pigment epithelium in hyperglycemia. Curr. Eye Res. 2012, 37, 1045-1053. [CrossRef] [PubMed]

23. Ye, E.A.; Liu, L.; Steinle, J.J. miR-15a/16 inhibits TGF-beta3/VEGF signaling and increases retinal endothelial cell barrier proteins. Vis. Res. 2017, 139, 23-29. [CrossRef] [PubMed]

24. Ma, W.; Silverman, S.M.; Zhao, L.; Villasmil, R.; Campos, M.M.; Amaral, J.; Wong, W.T. Absence of TGF $\beta$ signaling in retinal microglia induces retinal degeneration and exacerbates choroidal neovascularization. eLife 2019, 8, e42049. [CrossRef]

25. Fisichella, V.; Giurdanella, G.; Platania, C.B.M.; Romano, G.L.; Leggio, G.M.; Salomone, S.; Drago, F.; Caraci, F.; Bucolo, C. TGF- $\beta 1$ prevents rat retinal insult induced by amyloid- $\beta$ (1-42) oligomers. Eur. J. Pharmacol. 2016, 787, 72-77. [CrossRef]

26. Dagher, Z.; Gerhardinger, C.; Vaz, J.; Goodridge, M.; Tecilazich, F.; Lorenzi, M. The Increased Transforming Growth Factor- $\beta$ Signaling Induced by Diabetes Protects Retinal Vessels. Am. J. Pathol. 2017, 187, 627-638. [CrossRef]

27. Tosi, G.M.; Orlandini, M.; Galvagni, F. The Controversial Role of TGF- $\beta$ in Neovascular Age-Related Macular Degeneration Pathogenesis. Int. J. Mol. Sci. 2018, 19, 3363. [CrossRef] 
28. Shen, W.; Lee, S.R.; Yam, M.; Zhu, L.; Zhang, T.; Pye, V.; Mathai, A.E.; Shibagaki, K.; Zhang, J.Z.; Matsugi, T.; et al. A combination therapy targeting endoglin and VEGF-A prevents subretinal fibro-neovascularization caused by induced müller cell disruption. Investig. Ophthalmol. Vis. Sci. 2018, 59, 6075-6088. [CrossRef]

29. Platania, C.B.M.; Maisto, R.; Trotta, M.C.; D'Amico, M.; Rossi, S.; Gesualdo, C.; D'Amico, G.; Balta, C.; Herman, H.; Hermenean, A.; et al. Retinal and circulating miRNA expression patterns in diabetic retinopathy: An in silico and in vivo approach. Br. J. Pharmacol. 2019, 176, 2179-2194. [CrossRef]

30. Martinez, B.; Peplow, P.V. MicroRNAs as biomarkers of diabetic retinopathy and disease progression. Neural Regen. Res. 2019, 41, 1858.

31. Jenkins, A.J.; Joglekar, M.V.; Hardikar, A.A.; Keech, A.C.; O’Neal, D.N.; Januszewski, A.S. Biomarkers in diabetic retinopathy. Rev. Diabet. Stud. 2015, 12, 159. [CrossRef] [PubMed]

32. Fong, D.S.; Aiello, L.; Gardner, T.W.; King, G.L.; Blankenship, G.; Cavallerano, J.D.; Ferris, F.L.; Klein, R. Retinopathy in Diabetes. Diabetes Care 2004, 27 (Suppl. S1), s84-s87. [CrossRef]

33. Bandello, F.; Toni, D.; Porta, M.; Varano, M. Diabetic retinopathy, diabetic macular edema, and cardiovascular risk: The importance of a long-term perspective and a multidisciplinary approach to optimal intravitreal therapy. Acta Diabetol. 2020, 57, 513-526. [CrossRef]

34. Gross, J.G.; Glassman, A.R.; Jampol, L.M.; Inusah, S.; Aiello, L.P.; Antoszyk, A.N.; Baker, C.W.; Berger, B.B.; Bressler, N.M.; Browning, D.; et al. Panretinal photocoagulation vs intravitreous ranibizumab for proliferative diabetic retinopathy: A randomized clinical trial. Jama-J. Am. Med Assoc. 2015, 314, 2137-2146. [CrossRef]

35. Maggio, E.; Sartore, M.; Attanasio, M.; Maraone, G.; Guerriero, M.; Polito, A.; Pertile, G. Anti-Vascular Endothelial Growth Factor Treatment for Diabetic Macular Edema in a Real-World Clinical Setting. Am. J. Ophthalmol. 2018, 195, 209-222. [CrossRef] [PubMed]

36. Huang, C.Y.; Lien, R.; Wang, N.K.; Chao, A.N.; Chen, K.J.; Chen, T.L.; Hwang, Y.S.; Lai, C.C.; Wu, W.C. Changes in systemic vascular endothelial growth factor levels after intravitreal injection of aflibercept in infants with retinopathy of prematurity. Graefe's Arch. Clin. Exp. Ophthalmol. 2018, 256, 479-487. [CrossRef]

37. Hirano, T.; Toriyama, Y.; Iesato, Y.; Imai, A.; Murata, T. Changes in plasma vascular endothelial growth factor level after intravitreal injection of bevacizumab, aflibercept, or ranibizumab for diabetic macular edema. Retina 2018, 38, 1801. [CrossRef]

38. Ang, W.J.; Zunaina, E.; Norfadzillah, A.J.; Raja-Norliza, R.O.; Julieana, M.; Ab-Hamid, S.A.; Mahaneem, M. Evaluation of vascular endothelial growth factor levels in tears and serum among diabetic patients. PLoS ONE 2019, 14, e0221481. [CrossRef]

39. Rini, B.I.; Michaelson, M.D.; Rosenberg, J.E.; Bukowski, R.M.; Sosman, J.A.; Stadler, W.M.; Hutson, T.E.; Margolin, K.; Harmon, C.S.; DePrimo, S.E.; et al. Antitumor activity and biomarker analysis of sunitinib in patients with bevacizumab-refractory metastatic renal cell carcinoma. J. Clin. Oncol. Off. J. Am. Soc. Clin. Oncol. 2008, 26, 3743-3748. [CrossRef]

40. Willett, C.G.; Boucher, Y.; Duda, D.G.; Di Tomaso, E.; Munn, L.L.; Tong, R.T.; Kozin, S.V.; Petit, L.; Jain, R.K.; Chung, D.C.; et al. Surrogate markers for antiangiogenic therapy and dose-limiting toxicities for bevacizumab with radiation and chemotherapy: Continued experience of a phase I trial in rectal cancer patients. J. Clin. Oncol. 2005, 23, 8136-8139. [CrossRef]

41. van Cutsem, E.; Paccard, C.; Chiron, M.; Tabernero, J. Impact of prior bevacizumab treatment on VEGF-A and PLGF levels and outcome following second-line aflibercept treatment: Biomarker post hoc analysis of the VELOUR trial. Clin. Cancer Res. 2020, 26, 717-725. [CrossRef] [PubMed]

42. Zehetner, C.; Bechrakis, N.E.; Stattin, M.; Kirchmair, R.; Ulmer, H.; Kralinger, M.T.; Kieselbach, G.F. Systemic counterregulatory response of placental growth factor levels to intravitreal aflibercept therapy. Investig. Ophthalmol. Vis. Sci. 2015, 56, 3279-3286. [CrossRef] [PubMed]

43. Li, Q.; Pang, L.; Yang, W.; Liu, X.; Su, G.; Dong, Y. Long non-coding RNA of myocardial infarction associated transcript (LncRNA-MIAT) promotes diabetic retinopathy by upregulating transforming growth factor- $\beta 1$ (TGF- $\beta 1$ ) signaling. Med Sci. Monit. 2018, 24, 9497. [CrossRef]

44. Kaneko, H.; Takayama, K.; Asami, T.; Ito, Y.; Tsunekawa, T.; Iwase, T.; Funahashi, Y.; Ueno, S.; Nonobe, N.; Yasuda, S.; et al. Cytokine profiling in the sub-silicone oil fluid after vitrectomy surgeries for refractory retinal diseases. Sci. Rep. 2017, 7, 1-9. [CrossRef] [PubMed] 
45. Khuu, L.A.; Tayyari, F.; Sivak, J.M.; Flanagan, J.G.; Singer, S.; Brent, M.H.; Huang, D.; Tan, O.; Hudson, C. Aqueous humour concentrations of TGF- $\beta$, PLGF and FGF-1 and total retinal blood flow in patients with early non-proliferative diabetic retinopathy. Acta Ophthalmol. 2017, 95, e206-e211. [CrossRef] [PubMed]

46. Ziller, N.; Kotolloshi, R.; Esmaeili, M.; Liebisch, M.; Mrowka, R.; Baniahmad, A.; Liehr, T.; Wolf, G.; Loeffler, I. Sex Differences in Diabetes- and TGF- $\beta 1$-Induced Renal Damage. Cells 2020, 9, 2236. [CrossRef]

47. Lane, P.H.; Snelling, D.M.; Babushkina-Patz, N.; Langer, W.J. Sex differences in the renal transforming growth factor- $\beta 1$ system after puberty. Pediatric Nephrol. 2001, 16, 61-68. [CrossRef]

48. Chatziralli, I.P. The Role of Glycemic Control and Variability in Diabetic Retinopathy. Diabetes Ther. 2018, 9 , 431-434. [CrossRef]

49. Fullerton, B.; Jeitler, K.; Seitz, M.; Horvath, K.; Berghold, A.; Siebenhofer, A. Intensive glucose control versus conventional glucose control for type 1 diabetes mellitus. Cochrane Database Syst. Rev. 2014, 2, CD009122. [CrossRef]

Publisher's Note: MDPI stays neutral with regard to jurisdictional claims in published maps and institutional affiliations.

(C) 2020 by the authors. Licensee MDPI, Basel, Switzerland. This article is an open access article distributed under the terms and conditions of the Creative Commons Attribution (CC BY) license (http://creativecommons.org/licenses/by/4.0/). 